\title{
Extracción de caninos mandibulares como opción de tratamiento
}

Herbert Miguel Orrego-Carrillo* Mcs. $_{1}$

Universidad Nacional Mayor de San Marcos, Lima, Perú

Recibido: 28 de agosto del 2016 Aprobado: 6 de octubre del 2016

*Autor de correspondencia: Herbert Miguel Orrego-Carrillo. Av. Las Palmeras 190 Dpto. 201 San Isidro. Lima. Perú. Teléfono: 5114223376 .Correo electrónico: herbertorrego@hotmail.com

Cómo citar este artículo: Orrego-Carrillo HM. Extracción de caninos mandibulares como opción de tratamiento. Rev Nac Odontol. 2017;13(24):79-86. doi: http://dx.doi.org/10.16925/od.v12i24.1661

Resumen. Introducción: la extracción de caninos se indica en casos en los cuales existe impactación de dichas piezas y las posibilidades de tracción ortodóntica son limitadas o nulas. Diagnóstico: se presenta el caso de un paciente de 36 años de edad que acude a consulta por no estar conforme con la estética de su sonrisa. Tratamiento: como alternativa de tratamiento en este caso, se realizaron extracciones de los caninos mandibulares en lugar de los primeros premolares. Discusión: fue posible mejorar las relaciones oclusales, el overjet y el overbite sin perjudicar el aspecto facial del paciente. Siempre que estratégicamente sea conveniente, los resultados funcionales y estéticos obtenidos nos sugieren la posibilidad de tener como opción esta alternativa en el tratamiento de nuestros casos. Conclusión: este esquema de extracciones permite entonces cumplir ambos objetivos: tanto el funcional como el estético; sin embargo, debería reservarse para los casos en los que sea ventajosa su aplicación, hasta que se realicen más pesquisas para garantizar sus efectos a largo plazo.

Palabras claves: extracción de caninos mandibulares, pacientes adultos, relaciones oclusales, tratamiento de ortodoncia. 


\title{
Extraction of mandibular canines as a treatment option
}

\begin{abstract}
Introduction: Removal of canines is indicated in cases where there is impact on these teeth and the possibilities of orthodontic traction are limited or zero. Diagnosis: It is the case of a 36-year-old patient who comes for consultation because he is not satisfied with the aesthetics of his smile. Treatment: As an alternative treatment in this case, mandibular canines were extracted instead of the first premolars. Discussion: It was possible to improve occlusal relations, overjet, and overbite without damaging the patient's facial appearance. Whenever strategically convenient, the functional and aesthetic results obtained suggest the possibility of having this alternative as an option in the treatment of our patients. Conclusion: This scheme of extractions then allows to fulfill both functional and aesthetic objectives; however, this should be reserved for cases in which its application is advantageous, until more research is done to guarantee its long-term effects.
\end{abstract}

Keywords: extraction of mandibular canines, adult patients, occlusal relations, orthodontic treatment.

\section{Extração de caninos mandibulares como opção de tratamento}

Resumo. Introdução: A extração de caninos é indicada em casos nos quais existe impactação de tais peças e as possibilidades de tração ortodôntica são limitadas ou nulas. Diagnóstico: Apresenta-se o caso de um paciente de 36 anos de idade que comparece à consulta por não estar de acordo com a estética de seu sorriso. Tratamento: Como alternativa de tratamento neste caso foram realizadas extrações dos caninos mandibulares no lugar dos primeiros pré-molares. Discussão: Foi possível melhorar as relações oclusais, o overjet e o overbite sem prejudicar o aspecto facial do paciente. Sempre que for estrategicamente conveniente, os resultados funcionais e estéticos obtidos nos sugerem a possibilidade de ter como opção essa alternativa no tratamento de nossos casos. Conclusão: Este esquema de extrações permite cumprir, então, ambos objetivos: tanto o funcional quanto o estético; porém, deveria ser reservado para os casos nos quais sua aplicação seja vantajosa, até que sejam realizadas mais pesquisas para garantir seus efeitos a longo prazo.

Palavras-chave: extração de caninos mandibulares, pacientes adultos, relações oclusais, tratamento de ortodontia. 


\section{Introducción}

La extracción de caninos, según la costumbre, se indica en casos en los cuales existe impactación de dichas piezas y las posibilidades de tracción ortodóntica son limitadas o nulas. Este procedimiento, por supuesto, tiene su escenario natural en el maxilar superior. La impactación de caninos es más frecuente en el género femenino $[1,2]$, se verifica más unilateral antes que bilateral, y el lado izquierdo se ve afectado levemente más que el derecho [3]. Adicionalmente, se puede señalar que es más frecuente que la pieza impactada se encuentre por palatino antes que por vestibular [4].

Sin embargo, la literatura no reporta la exodoncia de caninos mandibulares como una opción frecuente de tratamiento. Las piezas a elegir más comúnmente son los premolares, seguidos en tasas muy lejanas por incisivos, e incluso algunas veces por molares.

Los caninos son piezas que tienen un rol muy importante, tanto estético, como funcional. Este concepto - sobretodo el estético- se ha enfatizado para el canino superior; se indica que su adecuada ubicación, forma y tamaño, permite generar un arco de sonrisa estéticamente deseable, ya que la eminencia canina da soporte al ala de la nariz y al labio superior. Si bien su rol en los movimientos excéntricos es protagónico al guiar a la mandíbula en las desoclusiones [5], esto no se resalta en el caso de los caninos mandibulares.

Pese a estas premisas, nuestros criterios para decidir qué pieza extraer se aplican de manera similar para ambos maxilares.

\section{Diagnóstico}

Paciente de sexo masculino de 36 años de edad y raza blanca, quien acude a la consulta privada con el deseo de resolver el apiñamiento y mejorar su sonrisa.

Al exámen extraoral presenta un rostro dolicofacial, simétrico, con un tercio inferior de la cara aumentado, quintos faciales proporcionados, perfil convexo y mentón retrognático. Intraoralmente, presenta dentición permanente, relaciones molares de Clase I, relaciones caninas de Clase III, líneas medias desviadas ambas a la izquierda $(3 \mathrm{~mm}$ la superior, y $1 \mathrm{~mm}$ la inferior), overjet de $3 \mathrm{~mm}$, overbite $50 \%$, caninos mandibulares fuera del arco y ausencia clínica de la piezas 24, 38 y 48 (figuras 1 y 2). Cefalométricamente, encontramos una relación esquelética de Clase ir por retrusión mandibular,
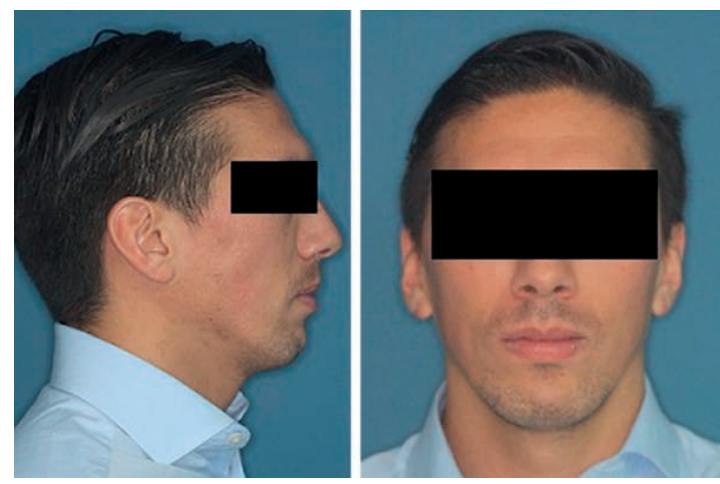

Figura 1. Fotografías externas-pretratamiento Fuente: elaboración propia
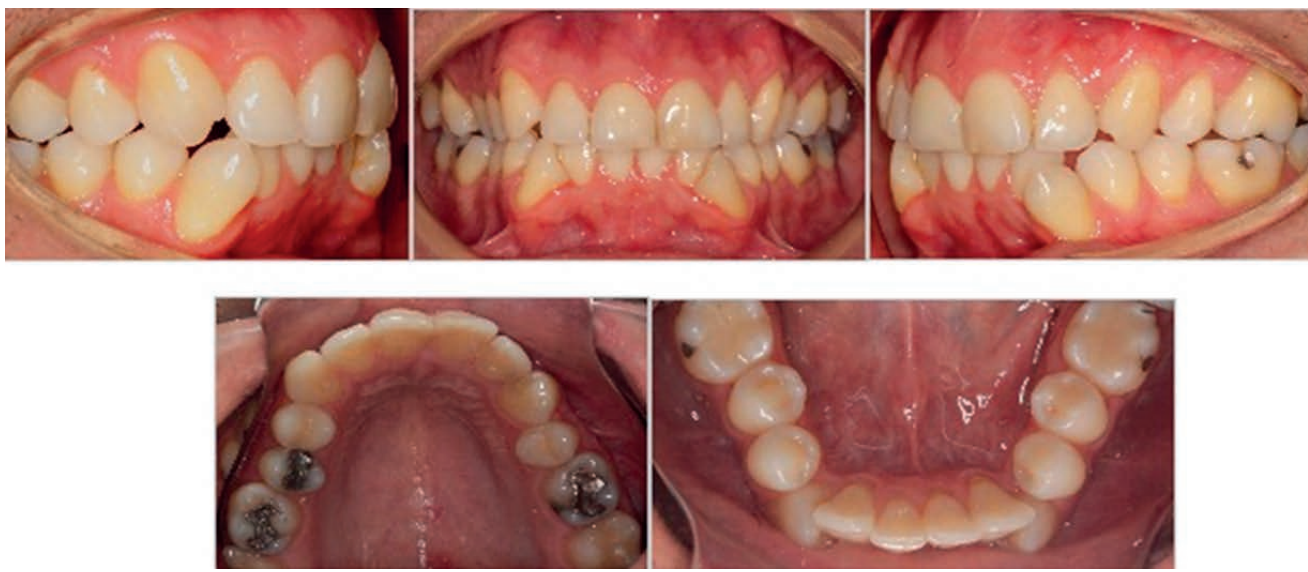

Figura 2. Fotografías internas-pretratamiento

Fuente: elaboración propia 
y un maxilar superior retroinclinado, incisivo superior vestibulizado y retruído, incisivo inferior vestibulizado y protruído; el paciente tuvo un crecimiento hiperdivergente. No presentaba signos ni síntomas de patología articular (figura 3).

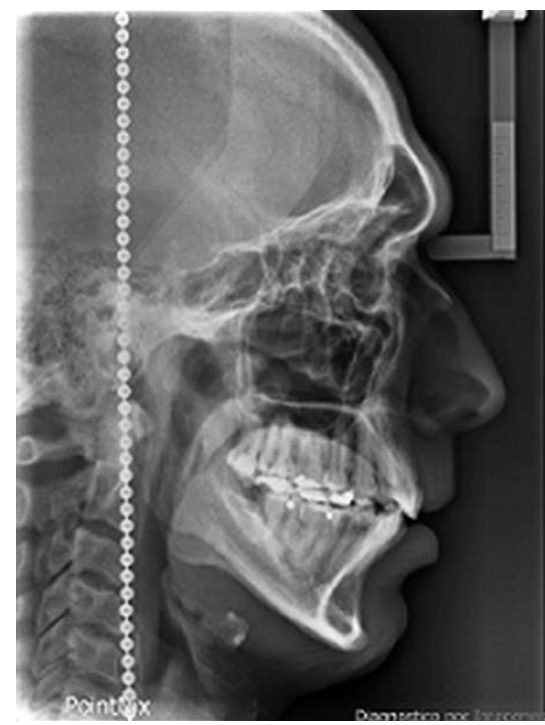

Figura 3. Radiografía cefalométrica pretratamiento Fuente: elaboración propia

La radiografía panorámica nos confirma la ausencia de las piezas 24,38 y 48 , lesiones cariosas múltiples y restauraciones coronarias diversas (figura 4).

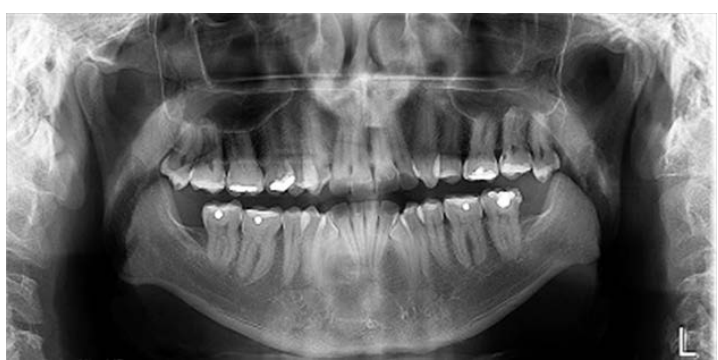

Figura 4. Radiografía panorámica pretratamiento Fuente: elaboración propia

\section{Objetivos de tratamiento}

Los objetivos del tratamiento involucraban preservar las relaciones molares de Clase I, aliviar el apiñamiento, mejorar las inclinaciones de los incisivos, corregir las líneas medias y generar una intercuspidación dentaria adecuada. Funcionalmente, conseguir que el paciente tuviera movimientos de lateralidad y protrusivos libres de interferencias. Estas metas deberían alcanzarse sin perjudicar el perfil del paciente.

Las decisiones de planificación biomecánica deben considerar el factor tiempo en todos los pacientes, pero especialmente en los pacientes adultos. En el presente caso, se decidió extraer la pieza 15 con el fin de permitir la corrección de la línea media superior y mejorar la inclinación de los incisivos. Las piezas 33 y 43 también fueron extraídas para construir una desoclusión mediada por los caninos superiores y por los primeros premolares inferiores; adicionalmente, esta decisión nos dio la oportunidad de mejorar la inclinación de los incisivos y corregir la línea media inferior.

\section{Alternativas de tratamiento}

Como opción terapéutica más frecuente para este caso, teníamos la exodoncia de los tres primeros premolares restantes [6]. Este esquema permitía a su vez la corrección de las relaciones molares y caninas, la línea media y el apiñamiento. Sin embargo, el traslado de los caninos inferiores era bastante amplio, dado que estas piezas ya se encontraban hacia la tabla vestibular, por lo cual su desplazamiento en algún momento enfrentaría la cortical, con el consiguiente riesgo para la integridad radicular. Además, este movimiento necesitaba mayor tiempo para completarse. En fin, el paciente decidió tomar la alternativa de la extracción de los caninos inferiores.

\section{Progreso del tratamiento}

Una vez realizadas las extracciones, se instaló aparatología preajustada de slot 0.022 en ambos maxilares, iniciamos la fase de alineamiento y nivelación con arcos de Nitinol superelástico 0,014 , luego 0,020 , y se finalizó esta fase con arcos de acero $0,019 \times 0,025$ coordinados (figura 5).

Antes de la fase de cierre de espacios hicimos la corrección de línea media con resortes de nitinol y gurines (figura 6). Una vez conseguido este objetivo empleando resortes de nitinol, se realizó el cierre de espacios (figura 7). Los acabados se trabajaron 
empleando arcos rectangulares trenzados de acero y elásticos intermaxilares. La radiografía panorámica de control (figura 8) nos permitió recolocar la aparatología, a fin de mejorar el paralelismo radicular fundamentalmente en el lugar de las extracciones inferiores.
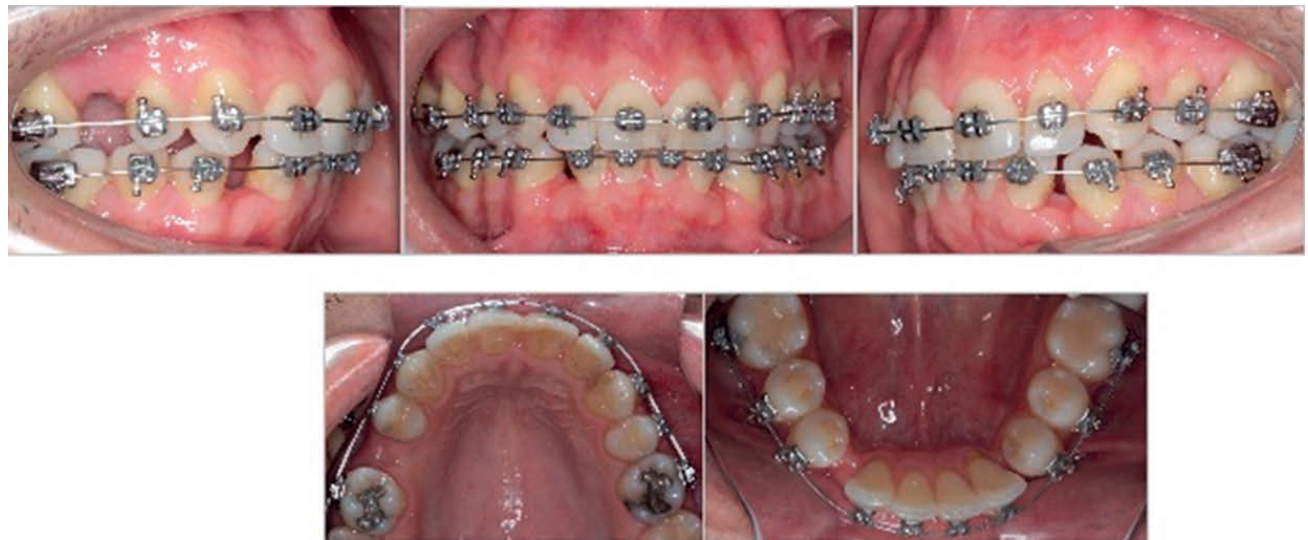

Figura 5. Alineamiento y nivelación

Fuente: elaboración propia
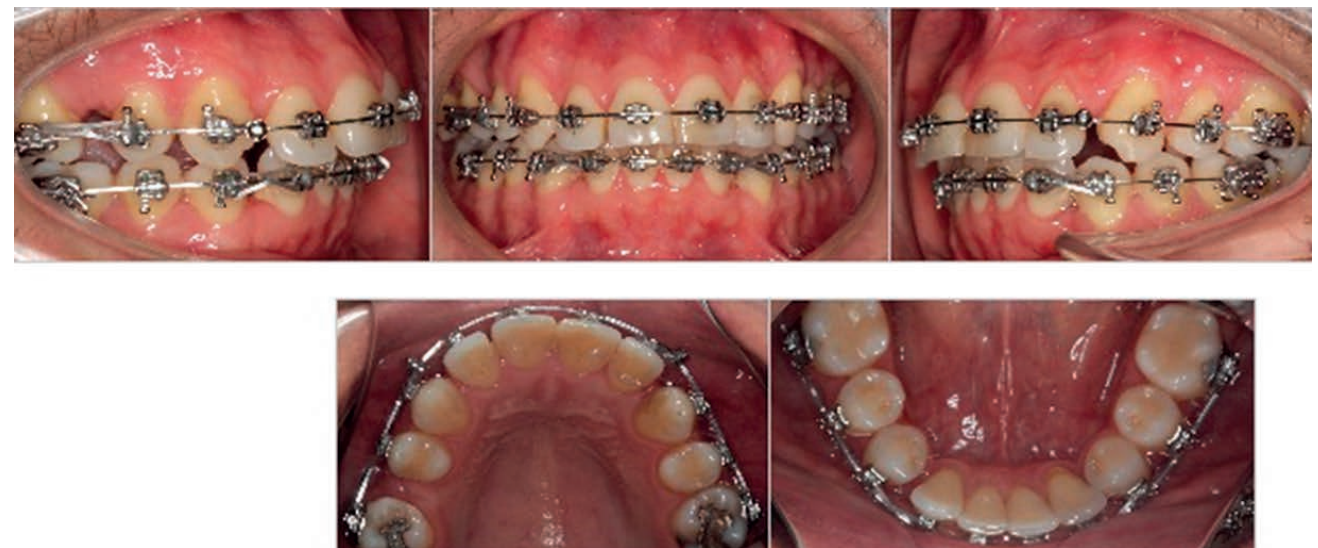

Figura 6. Corrección de línea media

Fuente: elaboración propia
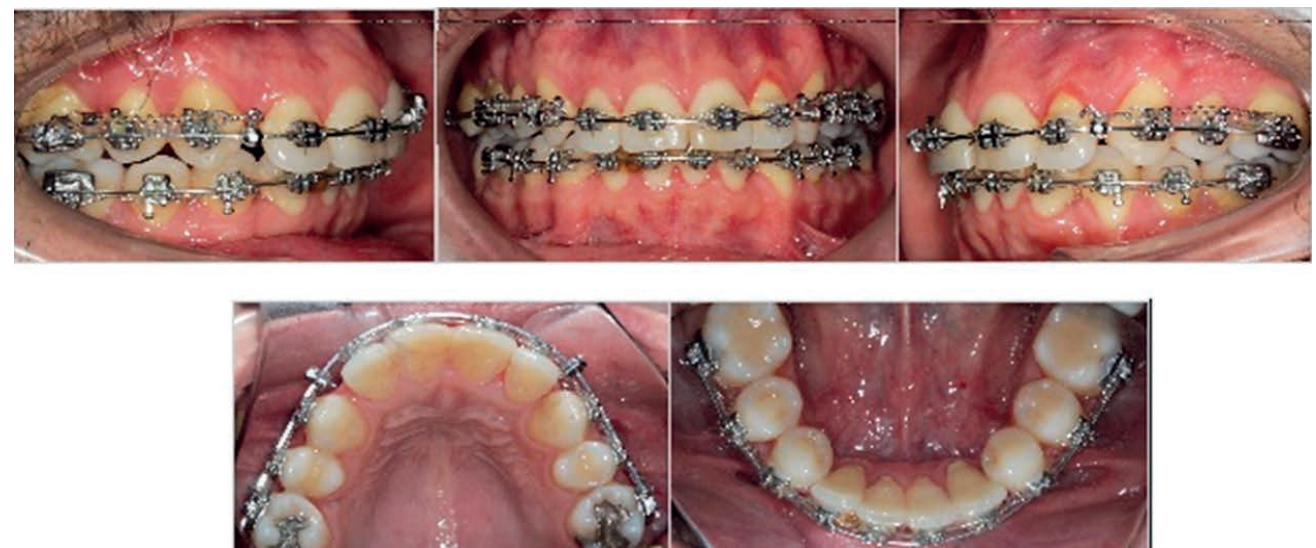

Figura 7. Cierre de espacios

Fuente: elaboración propia 


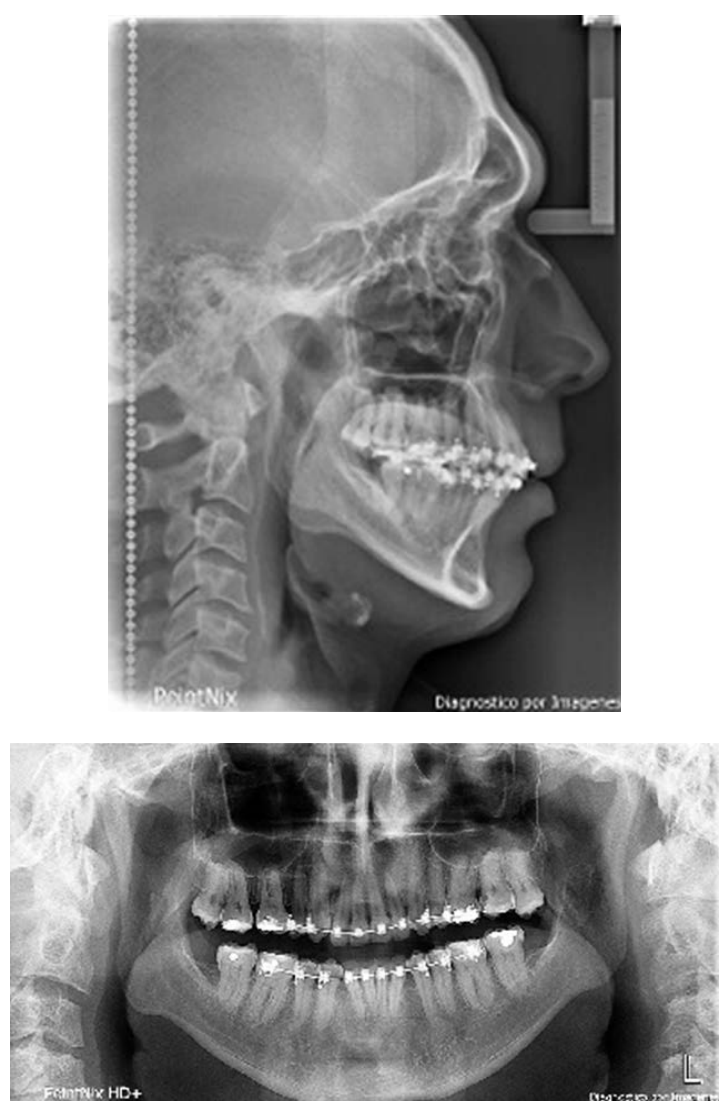

Figura 8. Radiografías cefalompetrucas y panorámicas de control

Fuente: elaboración propia

\section{Resultados del tratamiento}

Después de 24 meses de tratamiento, en cuanto al aspecto extraoral, el paciente mantuvo las características faciales que presentaba antes del mismo. Su perfil, pese a las extracciones, no sufrió modificaciones.

Se obtuvo un correcto alineamiento, así como una adecuada nivelación. El overjet y el overbite estaban dentro de los rangos normales, se consigueron relaciones molares y caninas de Clase I, la línea media tenía una discrepancia de $1 \mathrm{~mm}$, y la sonrisa era agradable (figuras 9 y 10).
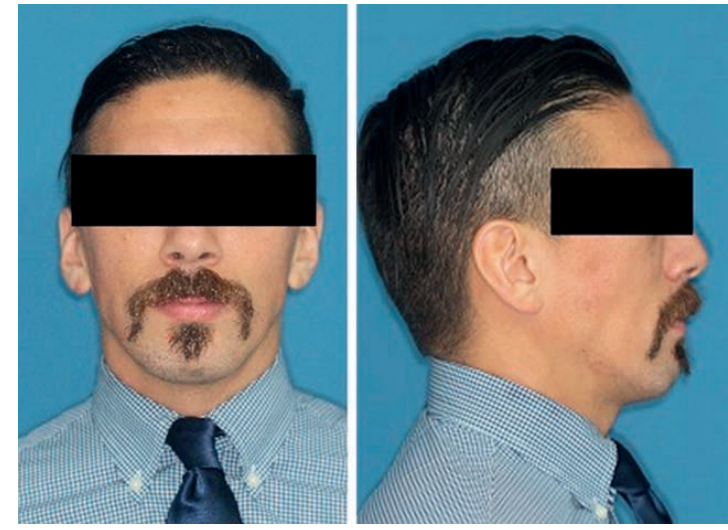

Figura 9. Fotografías externas postratamiento Fuente: elaboración propia
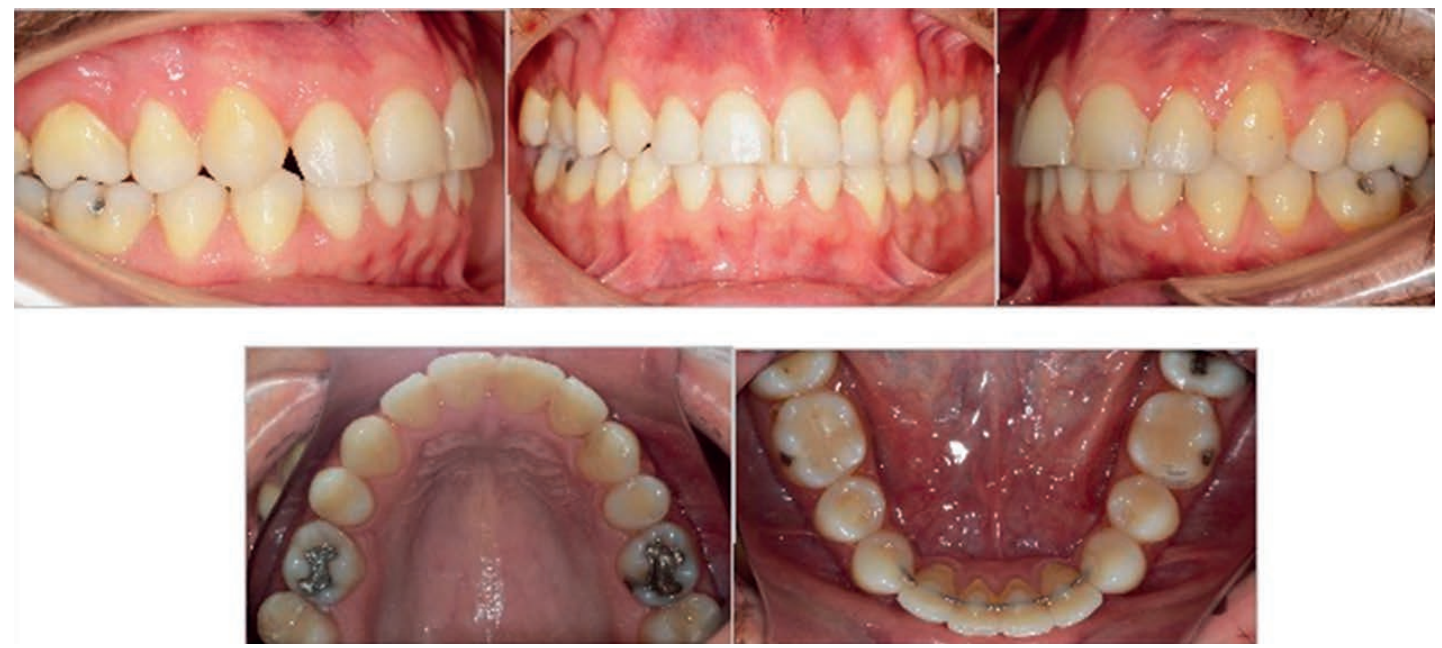

Figura 10. Fotografías internas postratamiento

Fuente: elaboración propia 


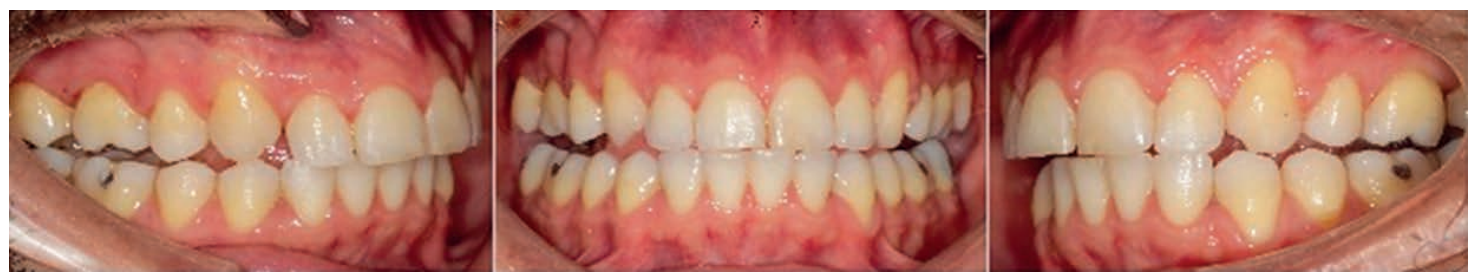

Figura 11. Movimientos de lateralidad y protrusiva, sin interferencias

Fuente: elaboración propia

Funcionalmente, se consiguió una protrusiva libre de interferencias y desoclusiones mediadas por el canino superior y las primeras premolares inferiores libres, así como de interferencias en el lado de no trabajo (figura 11).

\section{Discusión}

La posibilidad de extraer caninos inferiores en lugar de los premolares pasa por analizar si es que estas piezas pueden reemplazar, en función a sus características, los caninos en su rol durante los movimientos excéntricos.

El canino inferior tiene la orientación de su eje longitudinal hacia incisal, mesial y ligeramente a lingual, formando un ángulo de $2^{\circ}$ a $3^{\circ}$ con el plano frontal. Su erupción se verifica de los 11 a 12 años, y el cierre de su ápice entre los 12 y 14 . Tiene como longitud promedio $25 \mathrm{~mm}$, siendo el promedio para la corona de $10 \mathrm{~mm}$, y de 15 para la raíz.

El primer premolar inferior tiene una inclinación de $5^{\circ}$ con el plano frontal, de $3^{\circ}$ con el plano medio, y con el eje longitudinal hacia lingual y mesial $21 \mathrm{~mm}$ de longitud en promedio, de los cuales $7 \mathrm{~mm}$ corresponden a corona y 14 a raíz.

Anatómicamente, en cuanto a la raíz, prácticamente no tenemos mayores diferencias $(1 \mathrm{~mm})$. Las coronas difieren entre 2 a $3 \mathrm{~mm}$ [7]. Si consideramos que en muchas ocasiones - sobretodo en adultos - los caninos presentan desgastes a nivel de sus bordes, las diferencias no serían tan marcadas. La ubicación de los brackets o la realización de dobleces en los arcos permitiría, sin mucha dificultad, colocar los premolares ligeramente más extruídos que el resto de piezas, a fin de permitirle dirigir las excursivas laterales [8].

Desde el punto de vista estético, las superficies vestibulares de caninos y premolares inferiores no difieren tanto si las comparamos con sus homólogos superiores, siendo que la relación funcional se establece por el contacto entre la superficie palatina del canino superior y la vestibular del inferior (en este caso la premolar). De esta manera, el aspecto funcional estaría preservado, así como el estético (el cual depende en mayor medida del canino superior).

Históricamente, las premolares han sido siempre las piezas extraídas con mayor frecuencia en tratamientos de ortodoncia [9]. Proffit [10], en un estudio en el que se evalúan 6000 pacientes vistos en un período de 40 años en la Universidad de Carolina del Norte, indica cómo, durante todo este tiempo, las exodoncias diferentes a premolares estuvieron en un 15\% (aquí se involucrarían molares, incisivos y caninos). La literatura especializada no reporta la extracción de caninos mandibulares como esquema terapéutico.

En el presente caso, los premolares tenían una longitud radicular adecuada y estaban comparándolas con los caninos, mucho mejor ubicados en el arco. Estas consideraciones reducirían los movimientos y el tiempo del tratamiento en el arco mandibular, sin afectar los aspectos estéticos $y$ funcionales.

\section{Conclusiones}

Los objetivos de tratamiento en ortodoncia son funcionales y estéticos. La posibilidad de extraer caninos mandibulares cuando es estratégicamente adecuado es perfectamente viable, siempre que implique una ventaja en la resolución del caso y las condiciones anatómicas de las premolares sean las normales.

En el presente caso, fue posible conseguir relaciones molares de Clase I, obtener una relación 
entre premolares inferiores y caninos superiores funcional, con desoclusiones sin interferencias. Asimismo, se mejoró la línea media y se llegó a una intercuspidación dentaria satisfactoria, todo esto sin desmejorar el perfil del paciente.

Este esquema de extracciones permite entonces cumplir ambos objetivos: tanto el funcional, como el estético. Sin embargo, debería reservarse para los casos en los que sea ventajosa su aplicación, hasta que se realicen más pesquisas que garanticen sus efectos a largo plazo.

\section{Referencias}

[1] Peck S, Peck L, Kataja M. The palatally displaced canine as a dental anomaly of genetic origin. Angle Orthod. 1994;64:249-56.

[2] Baccetti T. A controlled study of associated dental anomalies. Angle Orthod. 1998;68:267-74.

[3] Leite H, de Oliveira S, de Araújo H. Labially displaced ectopically erupting maxillary permanent canine: Interceptive treatment and long-term results. Am J Orthod Dentofacial Orthop. 2005;128:241-51.
[4] Ericson S, Kurol J. Resorption of incisors after ectopic eruption of maxillary canines. A Ст study. Angle Orthod. 2000;70:415-23

[5] Abduo J, Tennant M, Mcgeachie J. Lateral occlusion schemes in natural and minimally restored permanent dentition: a systematic review. Journal of Oral Rehabilitation. 2013;40:788-802.

[6] Dardengo C, Fernandes L, Capelli J. Frequency of orthodontic extraction. Dental Press J Orthod. 2016; Jan-Feb,21(1):54-9.

[7] Esponda R. Anatomía Dental. 1ª reimpresión. México DF: Universidad Autónoma de México; 1994.

[8] Mirabella D, Giunta G, Lombardo L. Substitution of impacted canines by maxillary first premolars: A valid alternative to traditional orthodontic treatment. Am J Orthod Dentofacial Orthop. 2013;143:125-33.

[9] Bradbury AJ. A current view on patterns of extraction therapy in British health service orthodontics. Br Dent J. 1985; Jul 20,159(2):47-50.

[10] Proffit, W. Forty-year review of extraction frequencies at a University orthodontic clinic. Angle Orthodontist. 1994;6:407-14. 